\title{
OSTECTOMÍA EN CUÑA PARA EL MANEJO QUIRÚRGICO DE LA DEFORMIDAD ANGULAR RADIO-ULNAR DISTAL
}

\section{WEDGE OSTECTOMY FOR SURGICAL CORRECTION OF DISTAL RADIO- ULNAR ANGULAR DEFORMITY}

\author{
J. IGNACIO ARIAS F. ${ }^{1}$, M.V., PhD \\ ${ }^{1}$ Investigador de Post Doctorado, ICBM, Facultad de Medicina, Universidad de Chile. \\ joarias@uchile.cl
}

\begin{abstract}
Angular deformities of the forelimb are relatively common problems in dogs. At an early age, these are typically originated by traumatism that cause premature closure of the distal forearms growth plates. These growth deformities are characterized by a combination of antebrachial length deficit, angular and rotational malalignment, elbow incongruity, and carpal subluxation. Many surgical techniques and preoperative planning methods have been described to correct these deformities. In this case study, the use of a meticulous pre-planning, measurement of the degree of lateral deviation of the distal radial epiphysis and established the shape, location and depth of the wedge segment that was going to be removed to achieve alignment of the forearm in orthogonal radiographs, allowed a successful surgical resolution of the case with a return to function of the affected forelimb.
\end{abstract}

Keywords: antebrachial growth deformity, external skeletal fixation, radius, ulna, dog

\section{RESUMEN}

Las deformidades angulares de las extremidades anteriores son problemas relativamente frecuentes en los perros. A temprana edad, éstas son causadas normalmente por golpes que producen un cierre prematuro de las fisis de crecimiento del antebrazo distal. Estas deformidades 
del crecimiento se caracterizan por una combinación de deficiencias en la longitud del antebrazo, mala alineación angular y rotacional, incongruencia del codo y la subluxación del carpo. Muchas técnicas quirúrgicas y métodos de planificación preoperatorios se han descrito para corregir estas deformidades. En éste caso, que se comunica aquí, se realizó una acuciosa planificación previa, con la medición de los grados de desviación lateral de la epífisis distal del radio y se estableció la forma, ubicación y profundidad del segmento en cuña que se removería para lograr la alineación del antebrazo, utilizando radiografías ortogonales del miembro anterior derecho en proyecciones medio-lateral y cráneo-caudal. Estas permitieron una resolución quirúrgica satisfactoria del caso con una vuelta a la función del miembro afectado.

Palabras clave: deformidad del crecimiento, antebrazo, fijación esquelética externa, radio, ulna, perro

\section{INTRODUCCIÓN}

Las deformidades antero-braquiales (DAB) son las malformaciones más frecuentes que afectan las extremidades de los perros (Johnson y col. 1994). Varias causas de DAB se han descrito, incluyendo trauma de la fisis ante-braquial con retención de cartílagos de crecimiento, chondrodistrofia, deformidades genéticamente inducidas, enfermedades metabólicas y nutrición desequilibrada (Lau 1977, Ramadan 1878, Vandewater 1983, Fox 1984a y Hazewinkel 1995). Las deformidades de crecimiento se caracterizan por una combinación de deficiencias en la longitud del antebrazo, mala alineación angular y rotacional, incongruencia del codo y la subluxación del carpo.. Los efectos secundarios pueden incluir osteoartritis (OA) del codo y las articulaciones del carpo.

Además, la incongruencia del codo se ha asociado con la ocurrencia de fragmentación del proceso coronoideo medial (FCP), Osteocondritis Disecante (OCD) del cóndilo medial del 
húmero, no-unión del proceso ancóneo (UAP) e Incongruencia Articular (IA) (Kirberger 1998, Wind 1982 y Wind y col. 1986).

En un estudio de las deformidades de las extremidades anteriores en perros se describe que el 75\% de éstas se debieron a anomalías cubitales distales de la fisis (O’Brien y col. 1971). Tales anormalidades pueden ser atribuidas a una multitud de etiologías (O’Brien y col. 1971, Fox 1984a, Conzemius y col. 1994, Vaughan 1976 y Carrig 1983), siendo el trauma la causa más común de cierre temprano de los cartílagos de crecimiento distales (Fox 1984a). Muchas técnicas quirúrgicas y métodos de planificación preoperatorios se han descrito para corregir estas deformidades (Fox y col. 2006, Balfour y col. 2000, Theyse y col. 2005, Forell y col.1993, Dismukes y col. 2008, Fox 1984b, Ramadan y col. 1978 y Morgan y Miller 1994). Las cirugías correctivas por lo general implican la osteotomía con una técnica en cuña ya sea abierta o cerrada (Ramadan y col. 1978, Morgan y Miller 1994 y Henney y Gambardella 1989). La osteotomía en cúpula (“dome”) también ha sido descrita (Meola y col. 2008). Después de la corrección, la fijación se puede lograr ya sea con fijadores externos, internos o placas de osteosíntesis, dependiendo de factores del paciente y preferencia del cirujano.

Para estos efectos, se ha descrito una serie de métodos que combinan la localización de la deformidad y la cuantificación del centro de rotación de la angulación (CORA) (Paley y col. 1994) en combinación con la asistencia tridimensional por medio de modelación computacional 3D aplicado a la porción antero-braquial de los perros (Fox y col. 2006). Todo esto con el objetivo de lograr la corrección mecánica o anatómica del eje del hueso afectado para recuperar con parámetros relativamente normales (Fox y col. 2006). No obstante, en las condiciones más 
prevalecientes en el país, tales metodologías y equipamiento para uso veterinario no están ampliamente disponibles.

En la comunicación de éste caso quirúrgico se mostrará que una acuciosa planificación previa unida al uso de las potencialidades de las herramientas disponibles, permiten una resolución satisfactoria de estos casos.

\section{DESCRIPCIÓN DEL CASO}

\section{RESEÑA}

$\begin{array}{ll}\text { Nombre del Paciente: } & \text { “WilWil” } \\ \text { Especie: } & \text { Canino } \\ \text { Raza: } & \text { Pastor Australiano } \\ \text { Sexo: } & \text { Macho } \\ \text { Edad: } & 8 \text { meses } \\ \text { Peso: } & 19 \mathrm{Kg}\end{array}$

Diagnóstico:

RETENCIÓN DE CARTÍLAGOS DE CRECIMIENTO RADIOULNAR PREFERENTEMENTE LATERAL, DEL MIEMBRO ANTERIOR DERECHO.

El paciente es referido a principios de Octubre del 2008 presentando una claudicación del miembro anterior derecho (MAD) y desviación evidente a nivel de la articulación radio-carpal.

\section{ANAMNESIS}

El dueño del paciente manifestó que el paciente se había golpeado a los 4 meses de edad al rodar por la escalera de la casa. Desde ese episodio, su mano derecha comenzó paulatinamente a desviarse hacia lateral y a claudicar. Antiinflamatorios no esteroidales se administraron sin lograr la remisión de la claudicación. 


\section{EXAMEN ORTOPÉDICO}

Al examen en estática el paciente presenta una marcada desviación radio-carpal derecha con apoyo sólo de los cojinetes mediales, además de una marcada sifosis del segmento lumbo-sacral de la columna y aumento del volumen de la fisis distal del radio. Al examen en dinámica la claudicación es evidente con apoyo de un 20\% del MAD a la caminata y sin apoyo al trote, además de un acompañamiento de una rápida flexión dorsal del cuello al momento de apoyar este miembro.

A la palpación el paciente muestra contractura de la musculatura del cuello, dolor a la palpaciónpresión de la articulación lumbo-sacra, hipertrofia de la musculatura del hombro del miembro anterior izquierdo (MAI), hipotrofia de musculatura del hombro y brazo, dolor a la flexoextensión del codo y del carpo.

\section{EXAMEN RADIOGRÁFICO}

Se obtuvieron vistas radiográficas ortogonales del antebrazo bilateral en proyecciones mediolateral (ML) y cráneo-caudal (CC). En la radiografía se observa una desviación de aproximadamente $35^{\circ}$ del aplomo normal del radio distal derecho en la vista CC, sin signos de rotación axial (Fig. 1).

Además de lo anterior se observó una incongruencia de la articulación del codo en la proyección ML, dado por un crecimiento longitudinal insuficiente del radio, no llegando éste a completar la articulación produciéndose una incongruencia de $5 \mathrm{~mm}$ entre la superficie articular distal del humero y proximal del radio (Fig. 2).

Luego de trazar y hacer la cuantificación del centro de rotación de la angulación (CORA), se calculó la forma, dirección y magnitud de la cuña a ostectomizar sobre la radiografía ortogonal CC del MAD (Fig. 3). Por otra parte se trazó también el lugar y el tamaño de la ostectomía a 
realizar en la diafisis proximal de la ulna para lograr hacer congruente la articulación del codo (Fig. 4).

\section{TÉCNICA QUIRÚRGICA}

Mediante una aproximación medial de la fisis distal del radio, se expuso la zona a ostectomizar mediante debridación roma. Se colocó un clavo con hilo central de sobre medida en la epífisis distal del radio en dirección medio lateral perpendicular al eje mayor de esta (Fig. 5a).

Luego se tomaron medidas mediante una regla métrica de acero inoxidable esterilizada para marcar las líneas de inicio del corte de la cuña mediante una sierra ortopédica oscilante (Fig. 5b), buscando restablecer el aplomo del miembro derecho mediante ostectomía en cuña y fijación externa tipo II A 3-2. Luego de las radiografías post quirúrgicas se midieron nuevamente los ángulos radio-carpales, observándose que el ángulo de desviación se había reducido hasta llegar a menos de $5^{\circ}$ (Fig. 6).

En segundo lugar se abordó la incongruencia del codo provocando un desestresamiento de la diáfisis proximal de la ulna mediante una ostectomía total de $1 \mathrm{~cm}$ que incluía la desperiostización de la zona. Esta se realizó mediante una aproximación caudal de la porción proximal de la diáfisis ulnar. Luego de ésta, se procedió a colocar una aguja de Kirschner de 1,5mm como guía de cierre de la ostectomía (Fig. 7). Ambas intervenciones fueron cerradas con sutura absorbible en base a ácido poliglicólico de 3/0 de diámetro, en al menos 3 planos de sutura y finalizando con un cierre intradérmico cuticular contínuo. 


\section{POST QUIRÚRGICO}

El paciente fue inmediatamente derivado a fisioterapia y rehabilitación kinesiológica para su evaluación y tratamiento, mostrando una recuperación satisfactoria de las incisiones quirúrgicas dentro de los 7 primeros días.

El paciente logró cargar casi el 60\% del peso en el MAD luego de 2 semanas de realizada la intervención quirúrgica. Las radiografías controles del primer mes mostraron buena alineación y avance del radio hacia el codo (Fig. 8).

Los fijadores externos fueron retirados al mes y medio de realizada la intervención quirúrgica con un resultado satisfactorio de una claudicación leve con apoyo constante del miembro en dinámica y en estática.

\section{DISCUSIÓN}

Una limitación importante del informe de este caso es que el grado de claudicación del perro antes y después de la cirugía fue evaluado por un método clínico ortopédico y no con análisis de marcha digital que se considera el método de predilección (gold standard) para lograr evaluar con mayor objetividad el progreso de este paciente. Sin embargo, a falta del equipamiento adecuado para realizar análisis de marcha, es posible llegar a una aproximación del progreso clínico del paciente mediante la combinación de información radiográfica con la evaluación subjetiva de la claudicación por distintos observadores. Por lo tanto, para los efectos de la intervención reportada aquí, el éxito se definió por las mejoras radiográficas en alineación de las extremidades y la evaluación subjetiva de la claudicación por el ortopedista, la kinesióloga y los propietarios, aun cuando estas observaciones pueden ser fuente de diferentes grados de sesgo.

El paciente de este informe fue considerado un candidato para la cirugía debido a la severidad de la claudicación y la suposición de que ésta pudo haber empeorado sin este tratamiento. Las 
deformaciones angulares del miembro pueden dar lugar a distribuciones anormales de las fuerzas en las articulaciones adyacentes, desalineamientos conjuntos y, potencialmente, subluxación, los cuales pueden ser factores predisponentes o desencadenantes de artrosis, claudicación y signos de dolor (Kettelkamp y col. 1988). En el reporte de caso publicado por Dismukes y col. (2008) se llegó a la conclusión que la intervención quirúrgica realizada por ellos de la cabeza del radio puedo haber dado lugar a una pérdida de congruencia húmero-radio del paciente tratado. En el presente reporte se puso atención a ese hecho y se intervino antes de tener ese resultado desfavorable.

Como resultado del tratamiento del paciente de este informe, se concluye que el estudio adicional a priori de la planificación de la cirugía, resulta crucial para el manejo de la deformación grave del antebrazo. Estos métodos proporcionan información adicional para el diagnóstico y la evaluación de la severidad de la deformidad, incrementando el conocimiento del cirujano y su confianza, antes de la cirugía, promoviendo la eficiencia quirúrgica y, potencialmente, reduciendo la duración de la cirugía y la anestesia. De todas formas, esta manera de abordar el problema no debiera desalentar la idea de ir incorporando en la medida de lo posible, primero de manera experimental y luego extensivo a la práctica profesional, metodologías complementarias más precisas entre las que se destacan el análisis tridimensional mediante tomografía axial computarizada (Dismukes y col. 2008) en combinación con sistemas de prototipado para generar un modelo sólido tridimensional del segmento anatómico alterado (Crosse y Worth 2010). 


\section{REFERENCIAS}

BALFOUR RJ, BOUDRIEAU RJ, GORES BR. (2000). T-plate fixation of distal radial closing wedge osteotomies for treatment of angular limb deformities in 18 dogs. Vet Surg. 29:207-217.

CARRIG CB. (1983). Growth abnormalities of the canine radius and ulna. Vet Clin North Am Small Anim Pract. 13:91-115.

CONZEMIUS MG, SMITH GK, BRIGHTON CT, MARION MJ, GREGOR TP. (1994).Analysis of physeal growth in dogs, using biplanar radiography. Am J Vet Res. 5ㅡ:22-27.

CROSSE KR, WORTH AJ. (2010). Computer assisted surgical correction of antebrachial deformity. Vet Comp Orthop Traumatol. 23(5):354-61.

DISMUKES DI, FOX DB, TOMLINSON JL, ESSMAN SC. (2008). Use of radiographic measures and three-dimensional computed tomographic imaging in surgical correction of an antebrachial deformity in a dog. J Am Vet Med Assoc. 232(1):68-73.

FORELL EB, SCHWARZ PD. (1993). Use of external skeletal fixation for treatment of angular deformity secondary to premature distal ulnar physeal closure. J Am Anim Hosp Assoc. 29:460476.

FOX DB, TOMLINSON JL, COOK JL, BRESHEARS LM. (2006). Principles of uniapical and biapical radial deformity correction using dome osteotomies and the center of rotation of angulation methodology in dogs. Vet Surg. 35:67-77.

FOX SM. (1984a). Premature closure of distal radial and ulnar physes in the dog. Part I. Pathogenesis and diagnosis. Compend Contin Educ Pract Vet. 6:128-138.

FOX SM. (1984b). Premature closure of distal radial and ulnar physes in the dog. Part II. Treatment. Compend Contin Educ Pract Vet. $\underline{6}: 212-221$.

HAZEWINKEL HAW, SCHOENMAKERS I. (1995). Influence of protein, minerals and vitamin D on skeletal development of dogs.Vet Clin Nutr 2:93-99.

HENNEY LHS, GAMBARDELLA PC. (1989). Premature closure of the ulnar physis in the dog - a retrospective clinical study. J Am Anim Hosp Assoc. 25: 573-581.

JOHNSON JA, AUSTIN C, BREUR GJ. (1994). Incidence of canine appendicular musculoskeletal disorders in 16 veterinary teaching hospitals from 1980 through 1989. Vet Comp Orthop Traumatol. 7:56-69.

KETTELKAMP DB, HILLBERRY BM, MURRISH DE, HECK DA. (1988). Degenerative arthritis of the knee secondary to fracture malunion. Clin Orthop Relat Res. (234):159-169. 
KIRBERGER RM, FOURIE SL. (1998). Elbow dysplasia in the dog: pathophysiology, diagnosis and control. J S Afr Vet Assoc. 69(2):43-54.

LAU RE. (1977). Inherited premature closure of the distal ulnar physis. J Am Anim Hosp Assoc. 13:609-612.

MEOLA SD, WHEELER JL, RIST CL. (2008). Validation of a technique to assess radial torsion in the presence of procurvatum and valgus deformity using computed tomography: a cadaveric study. Vet Surg. 37:525-529.

MORGAN PW, MILLER CW. (1994). Osteotomy for correction of premature growth plate closure in 24 dogs. Vet Comp Orthop Traumatol. 7:129-135.

O'BRIEN TR, MORGAN JP, SUTER PF. (1971). Epiphyseal plate injury in the dog: a radiographic study of growth disturbance in the forelimb. J Small Anim Pract. 12:19-36.

PALEY D, HERZENBERG JE, TETSWORTH K, MCKIE J, BHAVE A. (1994). Deformity planning for frontal and sagittal plane corrective osteotomies. Orthop Clin North Am. 25(3):42565.

RAMADAN RO, VAUGHAN LC. (1978). Premature closure of the distal ulnar growth plate in dogs - a review of 58 cases. J Small Anim Pract. 19:647-667.

RAMADAN RO, VAUGHAN LC. (1978). Premature closure of the distal ulnar growth plate in dogs—a review of 58 cases. J Small Anim Pract. 19:647-667.

THEYSE LF, VOORHOUT G, HAZEWINKEL HA. (2005). Prognostic factors in treating antebrachial growth deformities with a lengthening procedure using a circular external skeletal fixation system in dogs. Vet Surg. 34:424-435.

VANDEWATER A, OLMSTEAD ML. (1983). Premature closure of the distal radial physis in the dog. A review of eleven cases. Vet Surg. 12:7-12.

VAUGHAN LC. (1976). Growth plate defects in dogs. Vet Rec. 98:185-189.

WIND AP, PACKARD ME. (1986). Elbow incongruity and developmental elbow diseases in the dog: Part II. J Am Anim Hosp Assoc. 22:725-730.

WIND AP. (1982). Incidence and radiographic appearance of fragmented coronoid process. Calif Vet 36:19-25.

WIND AP. (1986). Elbow incongruity and developmental elbow diseases in the dog: Part I. J Am Anim Hosp Assoc. 22:711-724. 


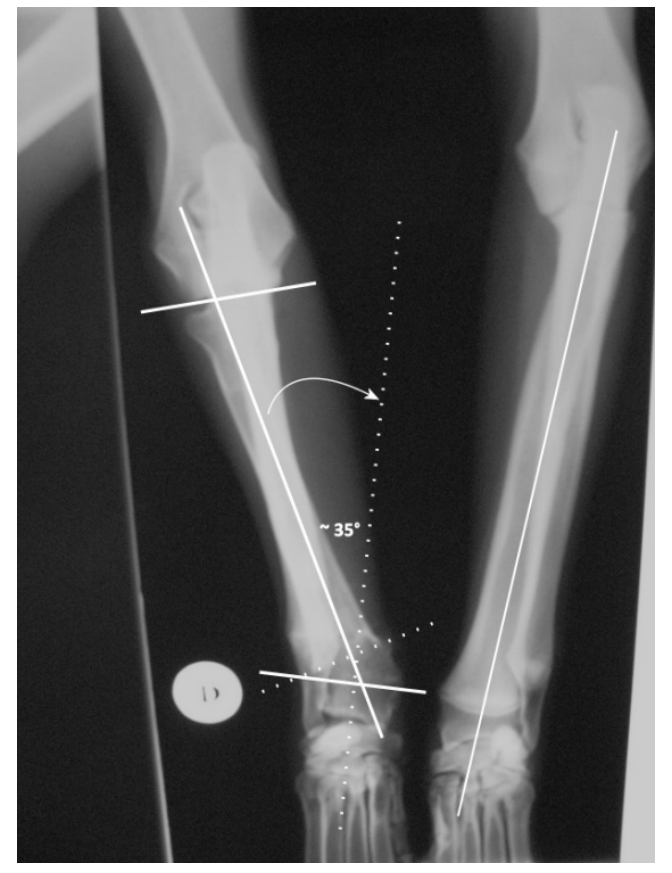

Figura 1. Vista cráneo-caudal de ambos antebrazos. Se muestran los $35^{\circ}$ de desviación desde el eje normal del radio derecho. También se muestra el eje normal del radio izquierdo.

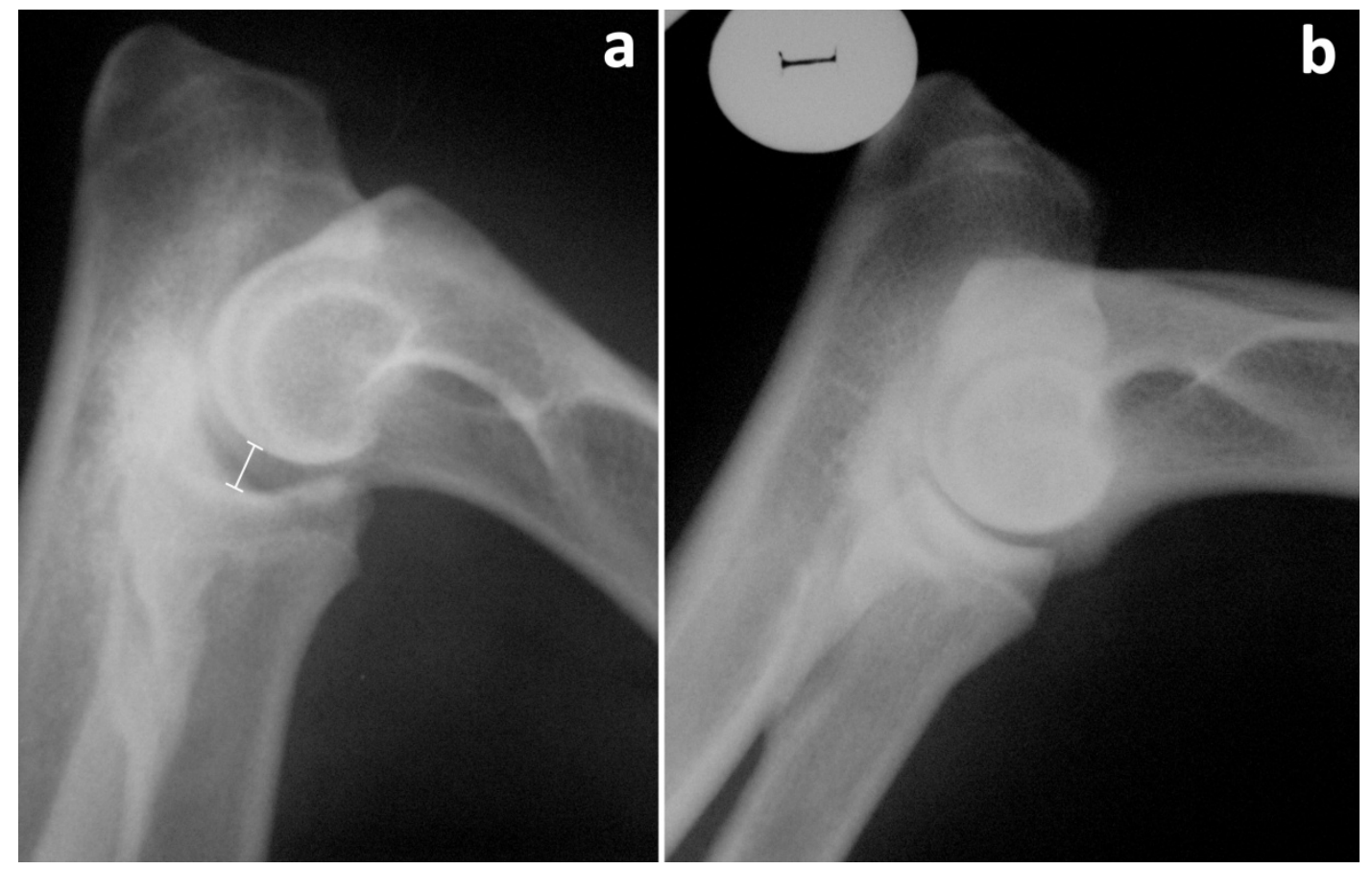

Figura 2. Vista medio lateral de la articulación del codo derecho (a) mostrando la incongruencia humeroradial de $5 \mathrm{~mm}$ (barra de tamaño) y el codo izquierdo (b) mostrando congruencia de la articulación. 


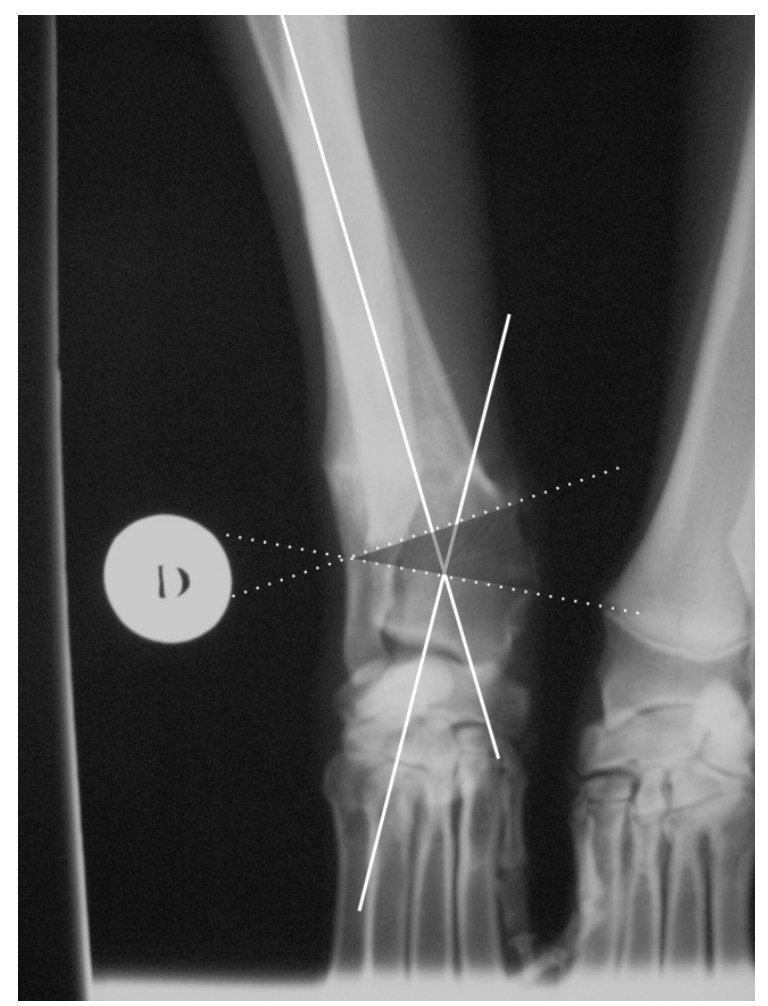

Figura 3. Vista cráneo-caudal de la fisis radial distal derecha mostrando en líneas punteadas el trozo de hueso radial a ser ostectomizado.

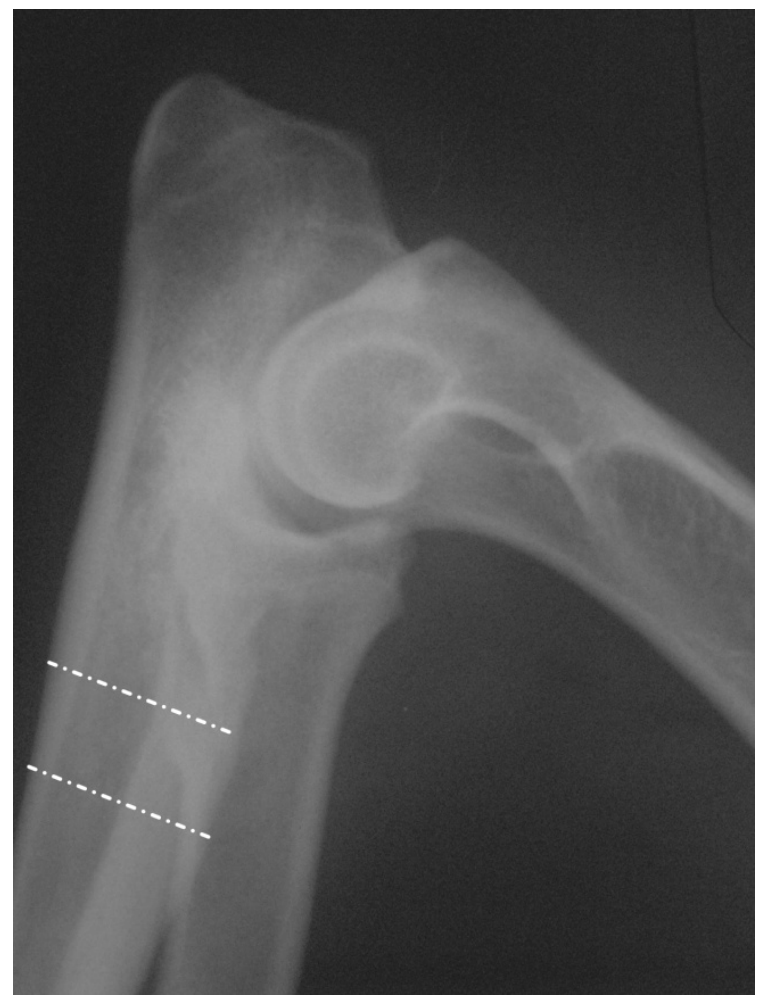

Figura 4. Vista medio-lateral de la ulna derecha mostrando en líneas punteadas el trozo de hueso de la diáfisis proximal a ser ostectomizado. 


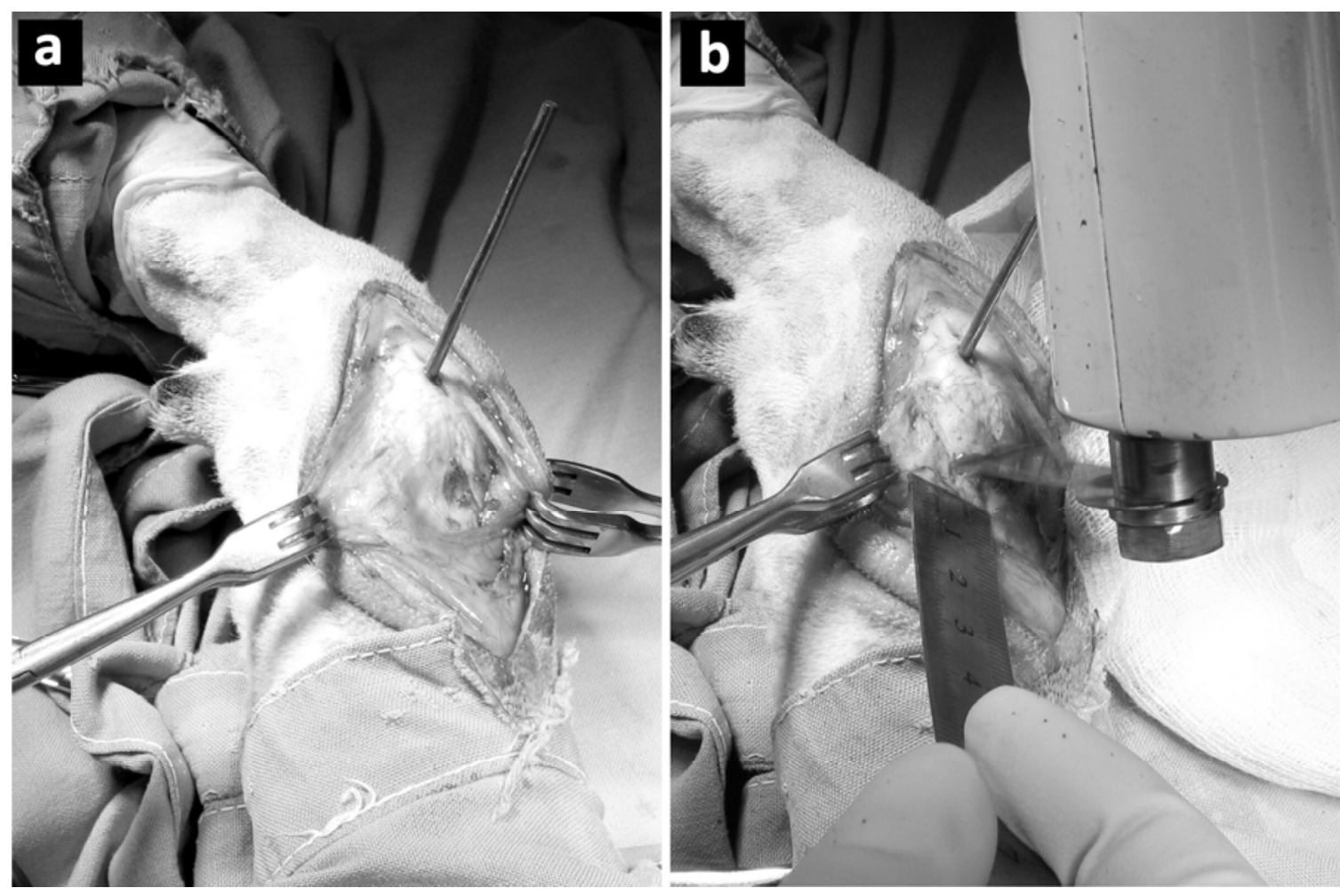

Figura 5. Fotografía de miembro anterior derecho con una aproximación medial de la fisis distal del radio y un clavo con hilo central de sobre medida en la epífisis distal del radio en dirección medio lateral perpendicular al eje mayor de ésta (a) y fotografía del inicio del corte en cuña del hueso a ostectomizar con una sierra oscilante (b).

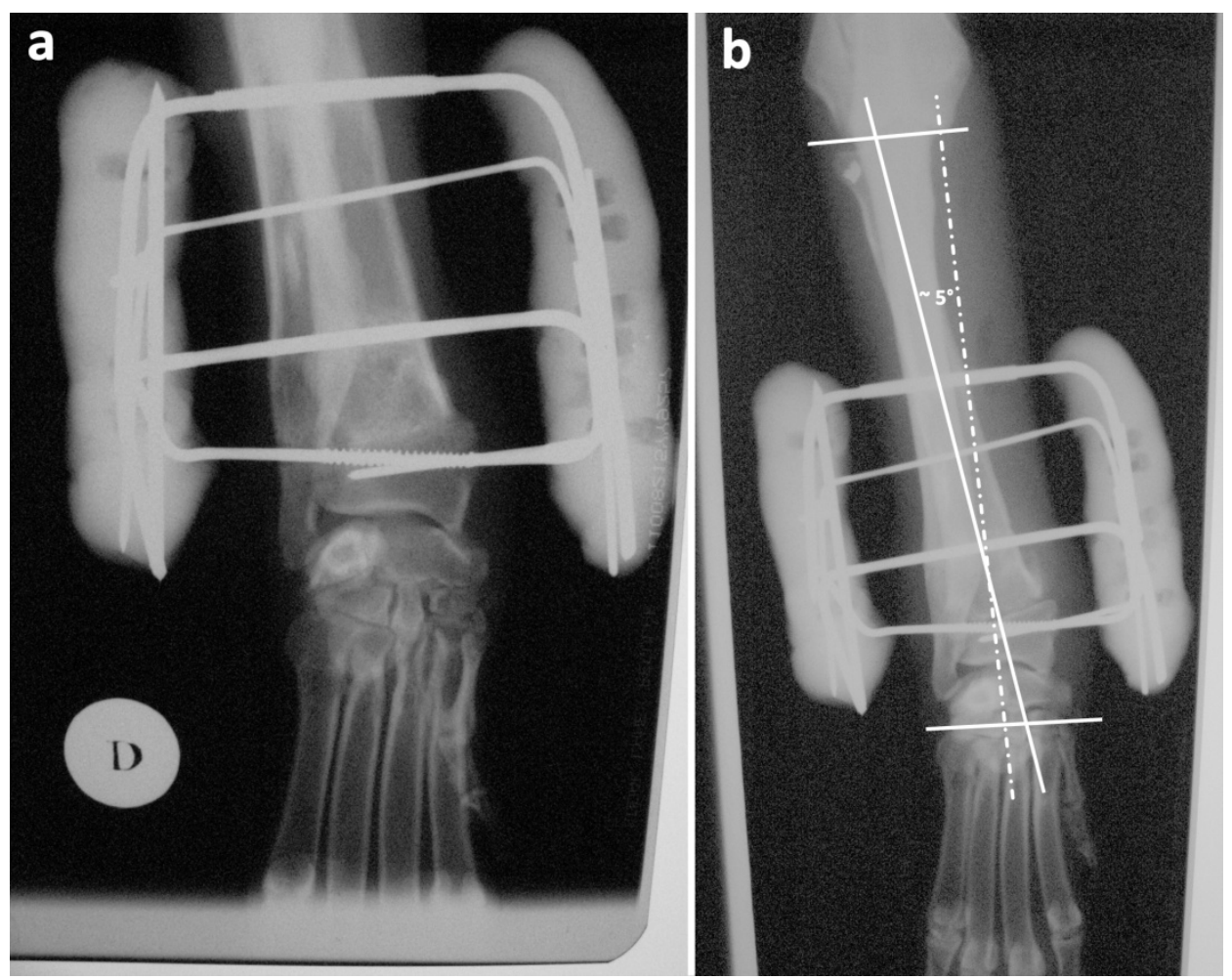

Figura 6. (a) Radiografía cráneo-caudal postquirúrgica de antebrazo de miembro anterior derecho con fijación externa II A 3-2 y (b) radiografía cráneo-caudal con las líneas de aplomo del radio (sólidas) y de la superficie articular distal del radio (punteada), nótese el ángulo de aproximadamente $5^{\circ}$ entre lo normal y lo logrado. 


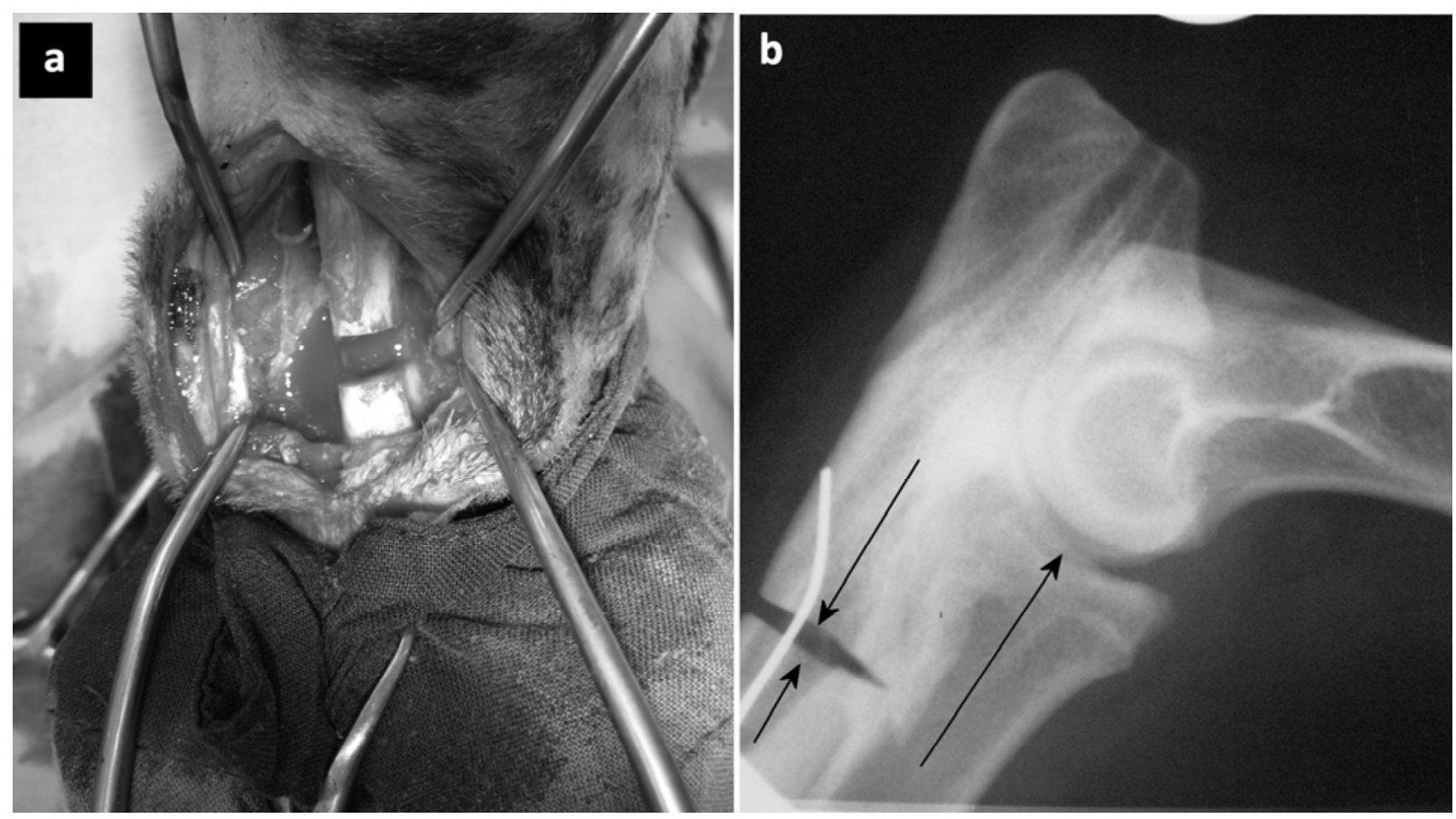

Figura 7. Fotografía de diáfisis proximal de miembro anterior derecho con trozo de $1 \mathrm{~cm}$ ostectomizado (a) y su radiografía de control postquirúrgico (b) con la aguja de Kirschner que guía su avance y cierre (flechas).

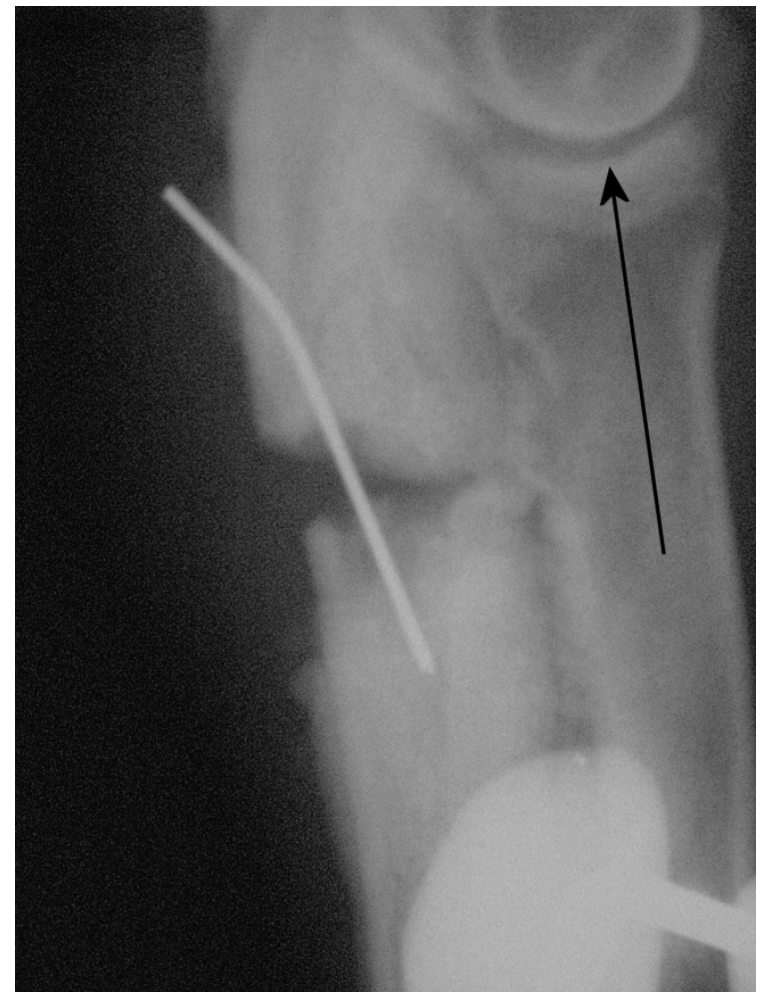

Figura 8. Radiografía control, vista medio-lateral de la porción diafisiaria proximal de la ulna derecha 1 mes postquirúrgico. Flecha muestra el avance que ha experimentado el radio hacia la articulación el codo. 